\title{
CINÉTICA DA DEGRADAÇÃO DA TEXTURA DE PIMENTAS EM CONSERVA
}

\author{
ANDRÉ S. DUTRA* \\ ANGELA APARECIDA L. FURTADO** \\ ROSIRES DELIZA*** \\ JOSÉ CARLOS S. FERREIRA****
}

\begin{abstract}
O objetivo deste trabalho foi avaliar o efeito do tratamento térmico (pasteurização) na cinética de degradação da textura de pimentas em conservas. Três cultivares de pimentas (CNPH 0053 - Pimenta Dedo-de-Moça, CNPH 0279 - Pimenta Escabeche e CNPH 0280 - Pimenta de Cheiro), produzidas pela Embrapa Hortaliças (em Brasília), foram avaliadas. As pimentas foram branqueadas por imersão em salmoura $(2 \% \mathrm{NaCl})$ a $100^{\circ} \mathrm{C}$ durante 1 minuto. Em seguida, foram envasadas em vidros e pasteurizadas a $100^{\circ} \mathrm{C}$ por $10,20,30$ e $40 \mathrm{~min}$, utilizando-se como líquido de cobertura salmoura acidificada com ácido acético $(2 \% \mathrm{NaCl})$. As amostras processadas foram analisadas quanto à firmeza e avaliadas quanto ao modelo cinético de degradação da textura. Os resultados revelaram que a textura dessas pimentas seguiu reação de $1^{\text {a }}$ ordem nos tempos de processamento da pasteurização analisados e o tempo de redução decimal (D) para as três pimentas em conserva encontrou-se abaixo de 100 minutos.
\end{abstract}

PALAVRAS-CHAVE: PASTEURIZAÇÃO; TEXTURA; CAPSICUM.

* Doutorando, Departamento de Tecnologia de Alimentos, Universidade Federal Rural do Rio de Janeiro (UFRRJ), Rio de Janeiro, RJ (e-mail: dutra_andre@yahoo.com.br).

** Engenheira Química, Pesquisadora, Embrapa Agroindústria de Alimentos, Guaratiba, Rio de Janeiro, RJ (e-mail: afurtado@ctaa.embrapa.br).

*** Engenheira de Alimentos, Pesquisadora, Embrapa Agroindústria de Alimentos, Guaratiba, Rio de Janeiro, RJ (e-mail: rodeliza@ctaa.embrapa.br).

**** Bacharel em Química, Analista, Embrapa Agroindústria de Alimentos, Guaratiba, Rio de Janeiro, RJ (email: zecarlos@ctaa.embrapa.br). 


\section{INTRODUÇÃO}

Todas as regiões brasileiras são produtoras e consumidoras de pimenta (Capsicum spp.), estimando-se a área de produção em 15.000 ha. A produção anual, aproximadamente 280.000 toneladas, destina-se tanto ao consumo in natura como ao processamento. O mercado anual, estimado em $\mathrm{R} \$ 80$ milhões, continua em crescimento pelo seu alto valor econômico. O agronegócio de pimentas tornou-se um dos mais importantes do País e um dos melhores exemplos de sucesso da agricultura familiar e de integração pequeno agricultor-agroindústria. As pimentas vermelhas respondem pelo terceiro lugar em produção e consumo de hortaliças para tempero no Brasil, perdendo apenas para o alho e a cebola (REIFSCHNEIDER et al., 2000).

As pimentas, além de serem consumidas in natura, podem ser processadas e utilizadas em diversas linhas de produtos na indústria de alimentos. Os frutos de pimentas picantes podem ser desidratados e comercializados inteiros, em flocos (calabresa), em pó (páprica picante - condimento), ou ainda em conservas, escabeches e molhos líquidos (FLAVOUR..., 2001).

Estudos sobre os métodos de conservação de pimentas são necessários para o ajuste dos parâmetros do processo de produção de conservas e estabelecimento de padrões de qualidade para os produtos industrializados.

A textura encontra-se entre os mais importantes atributos da qualidade de frutas e vegetais processados termicamente. A textura pode ser definida como um grupo de características físicas que surge dos elementos estruturais dos alimentos. São percebidas pelo sentido do tato e relacionadas com a deformação, desintegração e fluxo do alimento submetido a determinada força, que podem ser medidas objetivamente por funções de massa, tempo e distância (BOURNE, 1982; RIZVI e TONG, 1997; VU et al., 2004).

Segundo NI, LIN e BARRET (2005), as operações térmicas utilizadas na produção de conservas e vegetais congelados freqüentemente resultam em significante perda da integridade da textura. Segundo VU et al. (2004), alterações na textura de frutas e vegetais durante o processamento podem estar relacionadas com mudanças enzimática e não-enzimática da pectina. A degradação enzimática da pectina é catalisada por diferentes pectinases, como pectinametilesterase (PME) e poligalacturanase (PG). A degradação enzimática da pectina por PME e PG ocorre em duas etapas. Na primeira, a pectina é desmetoxilada pela PME resultando na produção de metanol, pectina com menor grau de metilação e ácido poligalacturônico. Posteriormente, ocorre a despolimerisação pela PG que resulta em pectina desmetoxilada com cadeia curta e como conseqüência mudança drástica na textura (amolecimento do tecido). VU et al. (2004) relataram que a pectina também pode ser degradada pela $\beta$-eliminação (via não-enzimática), reação química que ocorre em elevadas temperaturas e altera o valor nutricional, a textura, a cor e o sabor de produtos alimentares durante o processamento.

A aceitação de vegetais processados depende de inúmeros fatores, incluindo aparência, textura, sabor e valor nutricional. Vegetais processados que mantêm a firmeza e a crocância são altamente desejáveis porque os consumidores associam esses atributos de textura ao frescor do vegetal in natura (BOURNE, 2002; FILLION e KILCAST, 2002; SZCZESNIAK, 1988; NI et al., 2005).

Poucos estudos têm abordado a cinética da mudança de textura, embora represente importante componente de qualidade para a maioria dos alimentos (VU et al., 2004). Maximizar a qualidade e minimizar a perda de textura durante o processamento térmico requer conhecimento da cinética de degradação da textura, mediante parâmetros cinéticos. Esses podem fornecer subsídios para entender e predizer as mudanças que podem ocorrer durante o processamento (VU et al., 2004).

A firmeza, um dos mais importantes componentes do atributo textura, indica o quanto macio ficará o vegetal quando submetido ao aquecimento (VILLARREAL ALBA et al., 2004). ABBATEMARCO e RAMASWAMY (1994) relataram que os vegetais começam a amolecer e perder sua crocância e firmeza durante o processamento térmico, principalmente, devido ao colapso da estrutura celular e mudanças químicas na matriz polissacarídica da parede celular. 
O objetivo deste trabalho foi avaliar o efeito do tratamento térmico (pasteurização) na cinética de degradação da textura de três cultivares de pimenta em conservas.

\section{MATERIAL E MÉTODOS}

\subsection{MATÉRIA-PRIMA}

Foram utilizadas como matéria-prima três cultivares de pimenta, oriundas da Embrapa Hortaliças, localizada em Brasília: CNPH 0053 (Pimenta Dedo-de-Moça), CNPH 0279 (Pimenta Escabeche) e CNPH 0280 (Pimenta de Cheiro).

\subsection{METODOLOGIA}

\subsubsection{Processamento Térmico}

Efetuou-se o processamento das pimentas na planta-piloto de operações unitárias da Embrapa Agroindústria de Alimentos, localizada no Rio de Janeiro. As pimentas foram selecionadas (considerando-se o tamanho e a integridade física), lavadas, branqueadas a $100^{\circ} \mathrm{C}$ durante um minuto e acondicionadas em embalagens de vidro transparente. Completou-se o volume das embalagens com solução de salmoura acidificada com ácido acético $(2 \% \mathrm{NaCl})$ visando atingir $\mathrm{pH}<4,5$ (FURTADO et al., 2006). Os vidros foram levados ao túnel de exaustão, fechados hermeticamente e os produtos pasteurizados a $100^{\circ} \mathrm{C}$ por até 40 minutos com intervalos regulares de 10 minutos. A amostra in natura foi considerada como o tempo zero (Figura 1).

\section{FIGURA 1 - FLUXOGRAMA DE PROCESSAMENTO DE PIMENTAS EM CONSERVA}

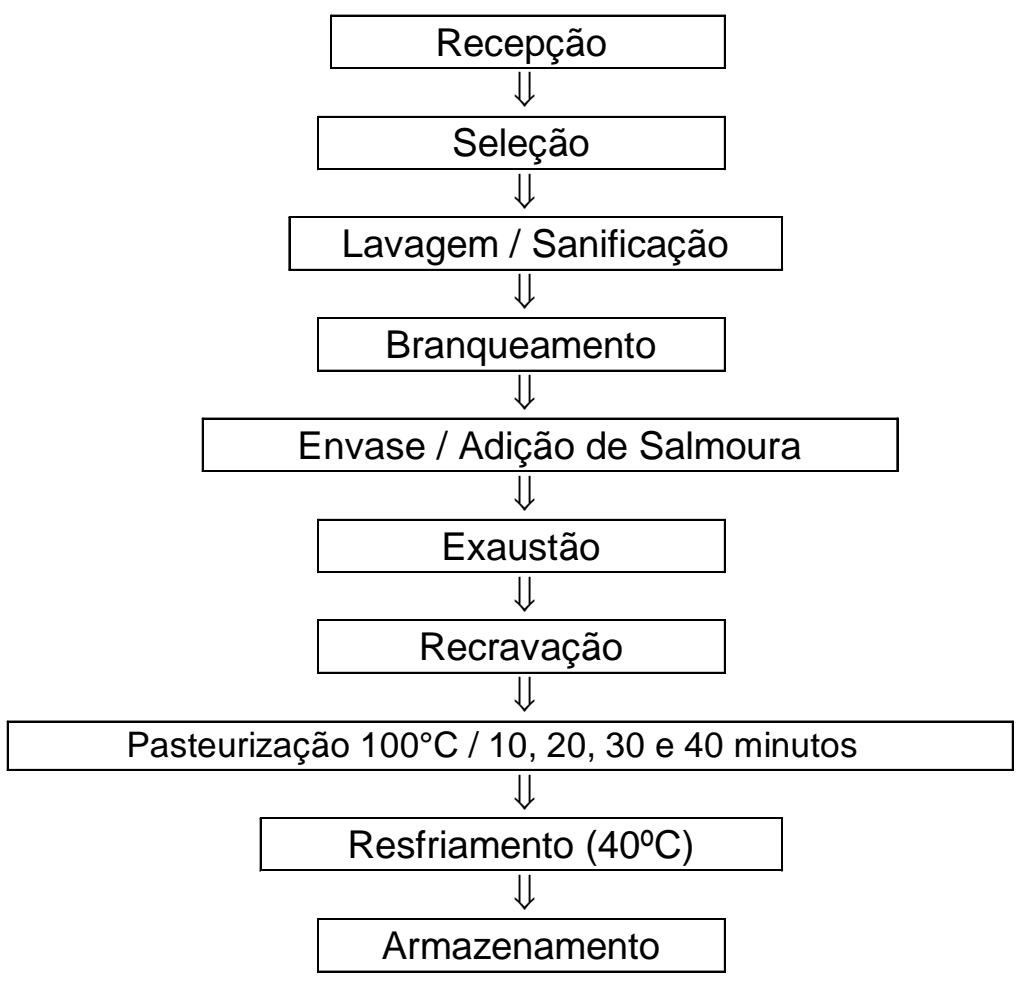




\subsubsection{Avaliação da Textura}

Após o processamento térmico, as conservas foram drenadas e encaminhadas para a determinação da firmeza. As extremidades das pimentas foram cortadas, os frutos abertos longitudinalmente e as sementes retiradas. A avaliação instrumental das amostras quanto à firmeza teve como parâmetro de controle pimentas in natura (tempo zero), as quais foram preparadas da mesma forma.

Usou-se texturômetro, modelo TA.Hdi (Stable Micro System, Goldalming, England), acoplado com probe cilíndrico de $2 \mathrm{~mm}$ de diâmetro para determinar a firmeza das pimentas por compressão. Operou-se o equipamento com força máxima da célula de carga de $50 \mathrm{~kg}$ nas seguintes condições: velocidade do pré-teste $2,0 \mathrm{~mm} / \mathrm{s}$; velocidade do teste $2,0 \mathrm{~mm} / \mathrm{s}$; velocidade do pós-teste $10,0 \mathrm{~mm} / \mathrm{s}$, distância de penetração de 1,0 mm e trigger tipo button. Fixou-se o índice de aquisição de dados em 200 pps, seguindo as recomendações do equipamento (STABLE MICRO SYSTEM, 1995). Analisou-se cada amostra separadamente com 10 repetições.

\subsubsection{Análise dos Dados}

Avaliaram-se os dados de firmeza no processamento térmico das três cultivares de pimentas em conserva segundo o modelo cinético de reações de $1^{\text {a }}$ ordem. Usou-se análise de regressão não-linear do programa Statistica (STATSOFT, 1998) em que a constante de velocidade da reação depende da concentração dos reagentes, conforme a equação 1.

$$
-d N / d t=k N
$$

Em que:

$$
\begin{aligned}
& N=\text { firmeza }(N) \\
& k=\text { constante de destruição térmica da textura }\left(\min ^{-1}\right) \\
& t=\text { tempo. }
\end{aligned}
$$

Integrando-se a equação 1 em relação ao tempo tem-se: $N=N_{0} \exp (-k t)$.

Forma bastante comum de representação do efeito da temperatura na velocidade das transformações em alimentos envolve o tempo de redução decimal (D) (TEIXEIRA NETO, VITALI e QUAST, 1991). A relação entre D e K pode ser representada, segundo FERREIRA et al.(1999), como: $k=2,303 / D$.

\section{RESULTADOS E DISCUSSÃO}

Avaliou-se a degradação térmica da textura (firmeza) das três cultivares de pimenta pela força de cisalhamento $(\mathrm{N})$. A análise de regressão não-linear de $\mathrm{N}$ versus $\mathrm{t}$ demonstrou que a textura das pimentas seguiu reação de $1^{\mathrm{a}}$ ordem nos tempos de processamento da pasteurização analisados. O modelo de reação de $1^{\text {a }}$ ordem pode ser visualizado nas Figuras 2 , 3 e 4 . Verificouse redução na firmeza, parâmetro de textura avaliado, conforme aumento no tempo de processamento. A Tabela 1 apresenta as equações obtidas dessas regressões e a Tabela 2 os parâmetros cinéticos $\mathrm{K}$ e $\mathrm{D}$, obtidos pelo processamento térmico.

Neste estudo, os resultados de degradação térmica da textura obtidos foram testados nos modelos de ordem zero, primeira ordem e segunda ordem. Porém, somente o modelo de primeira ordem descreveu o fenômeno observado com coeficientes de determinação (R2) mais próximos de 1 (modelo mais adequado). 
FIGURA 2 - DEGRADAÇÃO TÉRMICA DA TEXTURA DO CULTIVAR CNPH 0279 A $100^{\circ} \mathrm{C}$ POR 10, 20, 30 E 40 MINUTOS

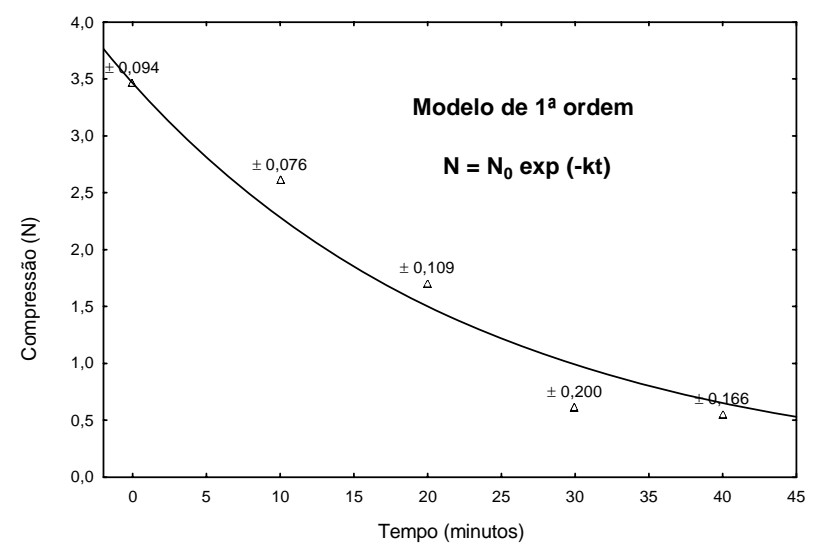

FIGURA 3 - DEGRADAÇÃO TÉRMICA DA TEXTURA DO CULTIVAR CNPH 0053 A $100^{\circ} \mathrm{C}$ POR 10, 20, 30 E 40 MINUTOS

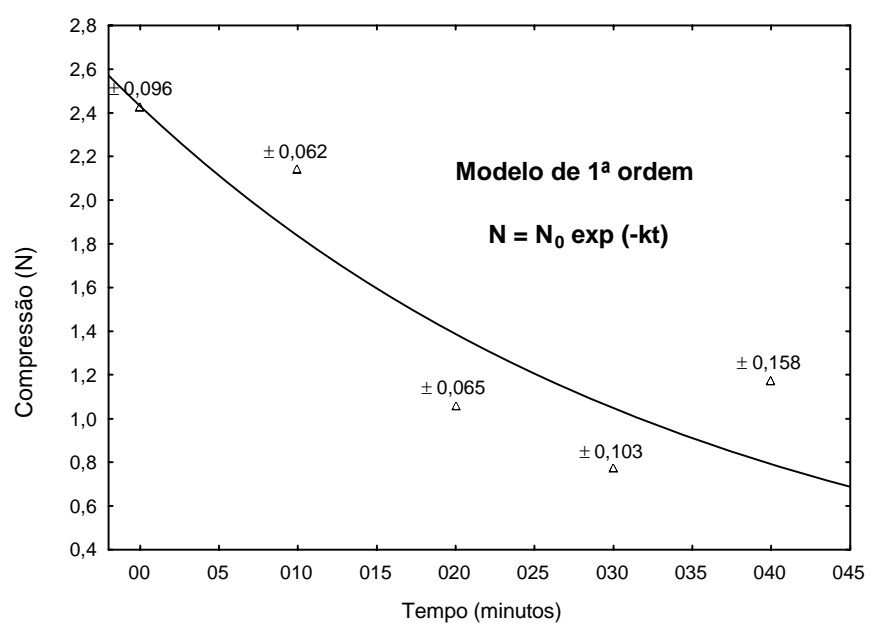

FIGURA 4 - DEGRADAÇÃO TÉRMICA DA TEXTURA DO CULTIVAR CNPH 0280 A $100^{\circ} \mathrm{C}$ POR 10, 20, 30 E 40 MINUTOS

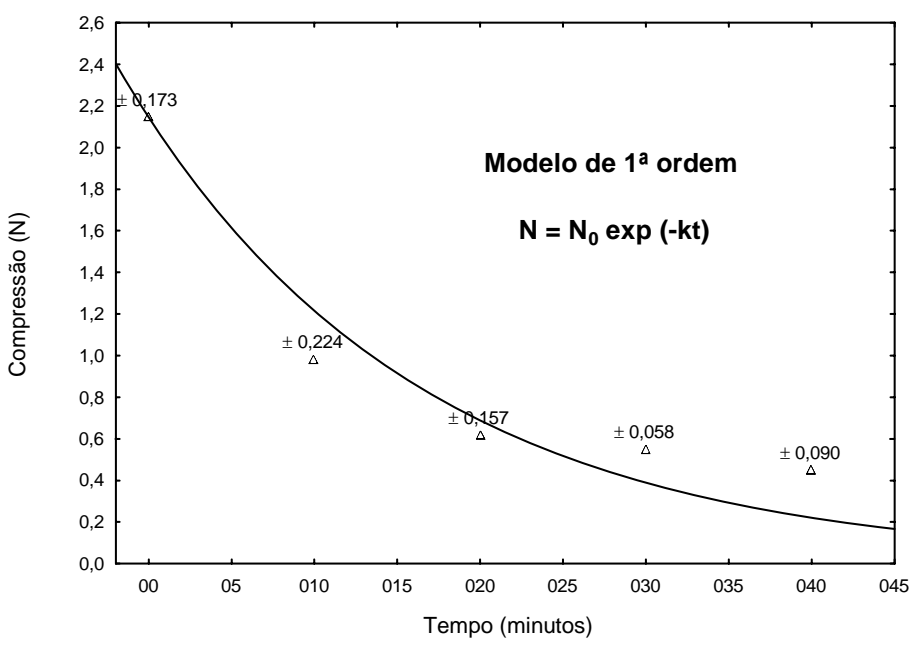


TABELA 1 - EQUAÇÕES OBTIDAS DA REGRESSÃO NÃO-LINEAR DE N VERSUS TEMPO PARA DEGRADAÇÃO DE TEXTURA DAS PIMENTAS EM CONSERVA

\begin{tabular}{|c|c|c|}
\hline Cultivar & Equação* & $\mathbf{R}^{2}$ \\
\hline CNPH 0279 & $\mathrm{~N}=3,464 \exp (-0,041764 \mathrm{t})$ & $0,976^{* *}$ \\
\hline CNPH 0053 & $N=2,431 \exp (-0,028047 t)$ & 0,894 \\
\hline CNPH 0280 & $N=2,145 \exp (-0,056843 t)$ & 0,964 \\
\hline \multicolumn{3}{|c|}{$\begin{array}{l}{ }^{*} \mathrm{~N}=\text { força de cisalhamento (Newton), } \mathrm{t}=\text { tempo (minutos). } \\
{ }^{* *} \text { Coeficiente de correlação. }\end{array}$} \\
\hline \multicolumn{3}{|c|}{$\begin{array}{c}\text { TABELA } 2 \text { - PARÂMETROS CINÉTICOS PARA A DEGRADAÇÃO TÉRMICA } \\
\text { DA TEXTURA DAS PIMENTAS EM CONSERVA }\end{array}$} \\
\hline Cultivar & $\begin{array}{c}\mathbf{k} \\
\left(\text { minutos }^{-1}\right)\end{array}$ & $\begin{array}{c}D \\
\text { (minutos) }\end{array}$ \\
\hline CNPH 0279 & 0,041764 & 55,143 \\
\hline CNPH 0053 & 0,028047 & 82,112 \\
\hline CNPH 0280 & 0,056843 & 40,515 \\
\hline
\end{tabular}

O tempo de redução decimal (D) para as três pimentas em conserva encontrou-se abaixo de 100 minutos para temperatura de tratamento de $100^{\circ} \mathrm{C}$. O valor de $\mathrm{D}$ obtido para textura das pimentas em conserva enquadrou-se nos fatores de qualidade em alimentos apresentados por LUND (1977).

LEE e HOWARD (1999) observaram decréscimo de 40\% na força de cisalhamento após a pasteurização de pimentas amarelas (Capsicum annuum), tratadas termicamente a $74^{\circ} \mathrm{C}$ por 10 minutos. LEE e HOWARD (1999) também constataram redução na firmeza das pimentas até 50 dias após a processamento, que se estabilizou entre 50 e 120 dias.

Neste estudo foi utilizado apenas o probe cilíndrico de $2 \mathrm{~mm}$ de diâmetro para a avaliação da firmeza das pimentas. Segundo BOURNE (1982) os resultados são afetados pelo probe e, no caso de pimenta, o recomendado é que se utilize o cilindro acima mencionado (STABLE MICRO SYSTEM, 1995).

\section{CONCLUSÃO}

Conclui-se que o aumento do tempo de processamento térmico de pasteurização das pimentas provoca alteração na textura, reduzindo a sua firmeza.

A degradação térmica da textura das pimentas em conserva seguiu reação de $1^{\circ}$ ordem nas faixas de tempo e temperatura estudadas. As constantes de velocidade de reação foram de 0,041764 $\mathrm{min}^{-1}$ para a CNPH 0279 (Pimenta Escabeche), 0,028047 $\mathrm{min}^{-1}$ para a CNPH 0053 (Pimenta Dedo-de-Moça) e 0,056843 $\mathrm{min}^{-1}$ para a CNPH 0280 (Pimenta de Cheiro). 


\begin{abstract}
KINETIC EVALUATION OF THE TEXTURE DEGRADATION CANNED PEPPERS

This study aimed at evaluating the thermal treatment effect (pasteurization) on the texture degradation kinetic of canned peppers. Three pepper species named as CNPH 0053 (Dedo-de-moça pepper), CNPH 0279 (Escabeche pepper), and CNPH 0280 (De cheiro pepper), all produced at Embrapa Hortaliças which is located in Brasília - DF (Brazil), were evaluated. Peppers were blanched by immersion in brine $(2 \% \mathrm{NaCl})$ at $100^{\circ} \mathrm{C}$ for $1 \mathrm{~min}$. After the bleaching the peppers were canned in glass bottle, and pasteurised at $100^{\circ} \mathrm{C}$ for zero, 10,20 , 30 , and $40 \mathrm{~min}$, using brine $(2 \% \mathrm{NaCl})$ with acetic acid as covering liquid. Firmness measures of all samples were taken, and afterwards, samples were analyzed regarding the texture degradation kinetic model. Results have shown that the pepper texture followed the first order reaction, considering the processing times used in the pasteurization. The parameter $\mathrm{D}$ (decimal reduction time) was below 100 minutes for the three investigated peppers.
\end{abstract}

KEY-WORDS: PASTEURIZATION; TEXTURE; CAPSICUM.

\title{
REFERÊNCIAS
}

1 ABBATEMARCO, C.; RAMASWAMY, H. S. End-over-end thermal processing of canned vegetables: effect on texture and color. Food Research International, v. 27, p. 327-334, 1994.

2 BOURNE, M. C. Food texture and viscosity: concept and measurement. New York: Academic Press, 1982. 325 p. (Food Science and Technology).

3 FERREIRA, V. L. P.; TEIXEIRA NETO, R. O.; MOURA, S. R.; SILVA, M. S. Cinética da degradação da cor de solução hidrossolúvel comercial de urucum, submetida a tratamentos térmicos. Ciência e Tecnologia de Alimentos, Campinas, v.19,n.1,1999. Disponívelem:<http://www.scielo.br/scielo.php?script=sci_arttext\&pid=S0101-20611999000 100010\&lng=pt\&nrm=iso\&tlng=pt>. Acesso em: 26 abr. 2006

4 FILLION, L.; KILCAST, D. Consumer perception of crispness and crunchiness in fruit and vegetables. Food Quality and Preference, v. 13, p. 23-29, 2002.

$5 \quad$ FLAVOUR and fragrance materials. Chicago: Allured Publishing Corporation, 2001. 215 p.

6 FURTADO, A. A. L.; DUTRA, A. S.; PACHECO, S.; OIANO NETO, J.; GODOY, R. L.O. Avaliação do efeito do tratamento térmico sobre a concentração de Beta-caroteno em pimenta (Capsicum) in natura e processada. In: CONGRESSO DE CIÊNCIA E TECNOLOGIA DE ALIMENTOS, 2., Curitiba, 2006. Curitiba: SBCTA, 2006. (CD-ROM).

7 LEE, Y.; HOWARD, L. Firmness and phytochemical losses in pateurized yellow banana peppers (Capsicum annuum) as affected by calcium chloride and storage. Journal Agricultural Food Chemistry, v. 47, p. 700-703, 1999.

8 LUND, D. B. Effect of heat processing on nutrients. In: HARRIS, R.S.; KARMAS, E. (Ed.). Nutritional evaluation of food processing. Westport, CT: Avi Publishing, 1975. Chap. 9, p. 205-243.

9 LUND, D. B. Design of thermal for maximizing nutrient retention. Food Techonology, v. 31, p. 71-78, Fev.1977.

$10 \mathrm{NI}$, L.; LIN, D.; BARRETT, D.M. Pectin methylesterase catalyzed firming effects on low temperature blanched vegetables. Journal of Food Engineering, v. 70, p. 546-556, 2005.

11 REIFSCHNEIDER, F.J.B. (Org.) Capsicum: pimentas e pimentões no Brasil. Brasília: Embrapa Comunicação para Transferência de Tecnologia / Embrapa Hortaliças, 2000. 113 p.

12 RIZVI, A. F.; TONG, C. H. Fractional conversion for determining texture degradation kinetics of vegetables. Journal of Food Science, v. 62, p. 1-7, 1997.

STABLE MICRO SYSTEM. Surface puncture strength of different coloured peppers using cylinder probe. Surrey,1995. 2 p. (Application Study. Ref. PEPP1/P3).

14 STATSOFT INC. Statistica for windows: release 5.1. Tulsa, USA, 1998.

16 processados. Campinas: ITAL, 1991. 36 p. (Manual Técnico, 6). 
17 VILLARREAL-ALBA, E.G.; CONTRERAS-ESQUIVEL, J.C.; AGUILAR-GONZÁLEZ, C.N.; REYES-VEJA, M.L. Pectinesterase activity and the texture of Jalapenõ pepper. Eur. Food Res. Technology, v. 218, p. 164-166, 2004.

18 VU, T. S.; SMOUT, C.; SILA, D. N.; LYNGUYEN, B.; VAN LOEY, A. M. L.; HENDRICKX, M. E. G. Effect of preheating on thermal degradation kinetics of carrot texture. Innovative Food Science and Emerging Technologies, v. 5, p. 37-44, 2004. 